Luján-Villar, J.D. (2018). Ciencias sociales y sostenibilidad: tecnologías de investigación social aplicadas a lo urbano y lo rural. Revista de Antropología y Sociología: VIRAJES, 20 (1). 61-81. DOI: 10.17151/rasv.2018.20.1.4

\title{
CIENCIAS SOCIALES Y SOSTENIBILIDAD: TECNOLOGÍAS DE INVESTIGACIÓN SOCIAL APLICADAS A LO URBANO Y LO RURAL*
}

\author{
JUAN DAVID LUJÁN-VILLAR*
}

Recibido: 30 de noviembre de 2017

Aprobado: 15 de diciembre de 2017

Artículo de Reflexión

\footnotetext{
* Agradezco al sociólogo Roberto Carlos Luján por sus oportunos comentarios y valiosa revisión conceptual. Los descargos de responsabilidad habituales se aplican.

** Magíster en Investigación Social Interdisciplinaria. Universidad Distrital Francisco José de Caldas, Bogotá, Colombia. E-mail: lujanvillar@gmail.com. ㅇ ORCID: 0000-0001-8622-4774. Google Scholar
} 


\title{
Resumen
}

Las tecnologías de investigación social son un conjunto de aplicaciones y modelos formales que posibilitan el abordaje de problemas sociales mediante métodos cuantitativos y cualitativos no necesariamente estadísticos, un gran campo iconológico de explicaciones visuales (Tufte, 1997), perspectivas transdisciplinares de conocimiento y ambientes de trabajo formal libres de disciplina. Este conjunto de aplicaciones presentan diversos retos y alternativas a los modelos mecánicos, estadísticos e interpretativos en estado puro de las ciencias sociales clásicas. El punto de partida de este trabajo enfatiza en la búsqueda de la sostenibilidad ecosistémica en escenarios urbanos y rurales y su investigación interdisciplinar, a propósito del impacto de las metodologías complejas, sus premisas, aplicaciones de trabajo práctico y conceptualizaciones básicas.

Palabras clave: sistemas complejos, modelado, simulación, sostenibilidad.

\section{SOCIAL SCIENCES AND SUSTAINABILITY: SOCIAL RESEARCH TECHNOLOGIES APPLIED TO THE URBAN AND THE RURAL}

\begin{abstract}
Social research technologies are a set of applications and formal models that allow the approach of social problems through quantitative and qualitative methods not necessarily statistical, a large iconological field of visual explanations (Tufte, 1997), transdisciplinary perspectives of knowledge and free from any discipline formal work environments. This set of applications presents different challenges and alternatives to the mechanical, statistical and interpretative models in the pure state of the classical social sciences. The starting point of this work emphasizes the search for ecosystem sustainability in urban and rural settings and its interdisciplinary research regarding the impact of complex methodologies, their premises, applications of practical work and basic conceptualizations.
\end{abstract}

Key words: complex systems, modeling, simulation, sustainability. 


\section{Complejidad y ciencias sociales en la búsqueda de la sostenibilidad}

n la búsqueda de modelos de sostenibilidad, algo se infiere de
modo sensato: jlos contextos si importan! Hace poco se publicó sobre la cuestión de la sostenibilidad poblacional. El artículo presentado por Nilsson, Griggs y Visbeck (2016) se preguntaba por la necesidad de establecer un mapeo de las interacciones entre los objetivos del desarrollo sostenible (sobre los 17 objetivos diseñados por las Naciones Unidas -NU— en su agenda presentada para 2030 en el año 2015) a través del reconocimiento de la interdependencia entre estos objetivos. Para ello los autores desarrollaron una escala de objetivos que influencian el desarrollo sostenible de una población con relación a la toma de decisiones a nivel político sobre posibles efectos (por ejemplo, del cambio climático).

Tabla 1. Escala de los siete objetivos de desarrollo sostenible y su interacción

\begin{tabular}{|c|c|c|c|}
\hline Interacción* & Nombre & Explicación & Ejemplo \\
\hline+3 & Indivisible & $\begin{array}{l}\text { Inextricablemente } \\
\text { conectado al éxito } \\
\text { de otro objetivo. }\end{array}$ & $\begin{array}{l}\text { Finalizar todas las formas } \\
\text { de discriminación contra las } \\
\text { mujeres y las niñas es indivisible } \\
\text { para asegurar la participación } \\
\text { total y completa de las mujeres } \\
\text { y las oportunidades de igualdad } \\
\text { en el liderazgo. }\end{array}$ \\
\hline+2 & Reforzando & $\begin{array}{l}\text { Ayuda el éxito de } \\
\text { otro objetivo. }\end{array}$ & $\begin{array}{l}\text { Al proveer electricidad se } \\
\text { refuerza las bombas de agua } \\
\text { y los sistemas de regadío. } \\
\text { Consolidando la capacidad de } \\
\text { adaptación relacionados al clima } \\
\text { se reducen las pérdidas causadas } \\
\text { por los desastres. }\end{array}$ \\
\hline+1 & Permitiendo & $\begin{array}{l}\text { Crea condiciones } \\
\text { para otro objetivo. }\end{array}$ & $\begin{array}{l}\text { Al proveer acceso a la } \\
\text { electricidad en hogares rurales se } \\
\text { posibilita la educación debido a } \\
\text { que hace posible realizar labores } \\
\text { en la noche con luz eléctrica. }\end{array}$ \\
\hline 0 & Consistente & $\begin{array}{l}\text { Interacciones } \\
\text { no significativas } \\
\text { positivas o } \\
\text { negativas. }\end{array}$ & $\begin{array}{l}\text { Asegurar la educación para } \\
\text { todos no interactúa de manera } \\
\text { significativa con el desarrollo } \\
\text { infraestructural o la conservación } \\
\text { de los ecosistemas oceánicos. }\end{array}$ \\
\hline
\end{tabular}




\begin{tabular}{|c|c|c|c|}
\hline-1 & Constreñido & $\begin{array}{l}\text { Limita las } \\
\text { opciones de otro } \\
\text { objetivo. }\end{array}$ & $\begin{array}{l}\text { Mejorar la eficiencia del agua } \\
\text { puede constreñir los regadíos } \\
\text { de la agricultura. Reduciendo } \\
\text { los cambios climáticos se puede } \\
\text { constreñir las opciones para el } \\
\text { acceso a la energía. }\end{array}$ \\
\hline-2 & Contrarrestando & $\begin{array}{l}\text { Disputa con otro } \\
\text { objetivo. }\end{array}$ & $\begin{array}{l}\text { AI impulsar el consumo para el } \\
\text { crecimiento se puede neutralizar } \\
\text { la reducción de las perdidas y la } \\
\text { mitigación del clima. }\end{array}$ \\
\hline-3 & Cancelando & $\begin{array}{l}\text { Hace imposible } \\
\text { alcanzar otro } \\
\text { objetivo. }\end{array}$ & $\begin{array}{l}\text { Asegurar por completo la } \\
\text { transparencia pública y la } \\
\text { responsabilidad democrática } \\
\text { no puede ser combinada con } \\
\text { los objetivos de la seguridad } \\
\text { nacional. La protección total de } \\
\text { las reservas naturales excluye el } \\
\text { acceso público a la recreación. }\end{array}$ \\
\hline
\end{tabular}

* La valoración de estas interacciones se establece en un rango en el cual +3 es el mayor valor positivo y -3 el mayor valor negativo. Fuente: adaptado de Nilsson, Griggs y Visbeck (2016).

El problema que los investigadores encuentran es que los tomadores de decisiones trabajan de manera independiente (por ejemplo, el ministerio de energía, salud o agricultura). Se hace necesario hacer coherente la relación entre política pública y sus estrategias de desarrollo, basadas en el conocimiento empírico sobre los modos mediante los cuales las intervenciones (públicas y privadas) afectan los objetivos de manera negativa o positiva (Nilsson, Griggs and Visbeck, 2016). En la tabla 1 se presenta una relación factible de las interacciones descritas, en apariencia sencilla pero integral, en la evaluación de proyectos sostenibles. El International Council for Science -ICSU- (2016) comenzó a implementar esta escala y su idea es popularizarla debido a su sencillez en el mapeo de interacciones entre el total de los 17 objetivos de desarrollo sostenible planteados por las NU.

El interés de los cientistas sociales por temáticas relacionadas con los modelos de desarrollo sostenible poblacionales, sus aspectos dinámicos y ecológicos es una posibilidad de conectar valores, conocimiento, puntos de vista y escenarios sociales (de Vries and Petersen, 2009). Con relación a la sostenibilidad, la noción de complejidad se puede comprender como mínimo de dos maneras. De Vries y Petersen (2009) consideran que la primera se puede establecer en términos cuantitativos, de diversidad y heterogeneidad, y las interacciones que producen los elementos del sistema abordado. La segunda — que se establece a partir de la extensiónexplica como el conocimiento incluye lo externo, la vida material versus 
lo interno, lo espiritual o el mundo de las ideas. El resultado de estas dos posiciones brindaría una complejidad agregada. Pero ¿por qué complejidad? Todo parece indicar que el colapso de algunos ecosistemas por tradición sostenibles puso en jaque muchas de las viejas premisas de la Modernidad (Northrop and Connor, 2013). El caso más emblemático quizá sea el de las fuentes de pesca en el noroeste del atlántico. En la pesca del bacalao del Atlántico (Gadus morhua) se capturaron 800000 toneladas de esta especie para 1965; pero en 1978 se inició una crisis sin precedentes, la cual tuvo su colapso y desplome hacia 1992 a casi cero toneladas (Northrop and Connor, 2013). Casi 500 años de buena pesca se desplomaron de un momento a otro. Todo parece indicar que las razones fueron la sobrepesca de la especie, una perspectiva no sostenible.

\section{Modelos basados en agentes (MBA) y dinámica de sistemas (DS)}

Algunos investigadores consideran que las agendas de sostenibilidad en un nivel social deben tener una correspondencia o balance entre la eficiencia y la resiliencia. Higgins et al. (2010) aplicaron metodologías sistémicas complejas (DS, MBA's y ARS) en el estudio de cadenas de valor de la agricultura como un medio para obtener información dinámica sobre el sistema. Debido a que un sistema complejo (SC) exhibe, por lo general, una dinámica no-lineal convirtiéndole en un modelo cambiante. La dinámica no-lineal ${ }^{1}$ hace parte de la teoría del caos y en este contexto explica cómo los sistemas pueden tener interruptores (switches) que hacen que el comportamiento de un sistema sea: estable, inestable, periódico y aperiódico en tanto modelo dinámico. Estas transiciones de fase o cambios abruptos producidos por las interrupciones son llamados puntos de inflexión (tipping points). Cualquier alteración en un input (entrada), parámetro o condición inicial desemboca en un cambio radical en su comportamiento final. De ahí que los sistemas alejados del equilibrio sean sensibles a las condiciones iniciales (Poincaré, 1908) y este tipo de sistemas sean estudiados desde el caos determinista.

\footnotetext{
${ }^{1}$ Este concepto es quizá la comprobación más relevante dentro de la teoría del caos. Las bifurcaciones son un ejemplo de este fenómeno. Una posible explicación sencilla radica en la forma mediante la cual comprendemos los sistemas vivos; se dice que un sistema posee no-linealidad cuando su comportamiento exhibe complejidad, caos, fenómenos susceptibles de ser visualizados mediante la graficación de atractores de punto fijo, atractores periódicos y atractores extraños. La idea central yace sobre la inestabilidad que presentan los sistemas incluyendo los sistemas sociales con una breve alteración de las condiciones iniciales entre los valores de parámetro y los valores de las variables (Holland, 1995; Reynoso, 2006).
} 
Concebir los fenómenos físicos y sociales más allá de sus premisas ontológicas como símiles establece un paralelo entre ambos tipos de sistemas. Debido a que en ambos casos las atribuciones a estos sistemas no son inherentes a ellos mismos, pues en determinados casos pueden exhibir las mismas características si consideramos que construir un modelo es una forma de comprender el mundo (Gilbert and Troitzsch, 2005). Tanto los niveles ontológicos, sistémicos y de simulación se encuentran en el plano de las ideas no en el nivel de las cosas mismas.

Pantalla

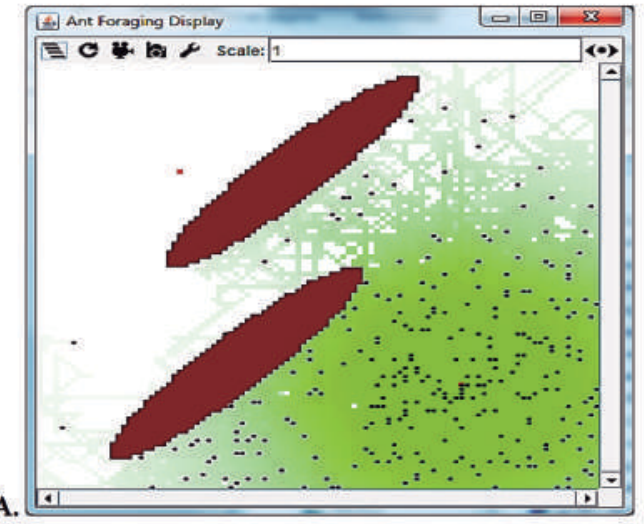

B.

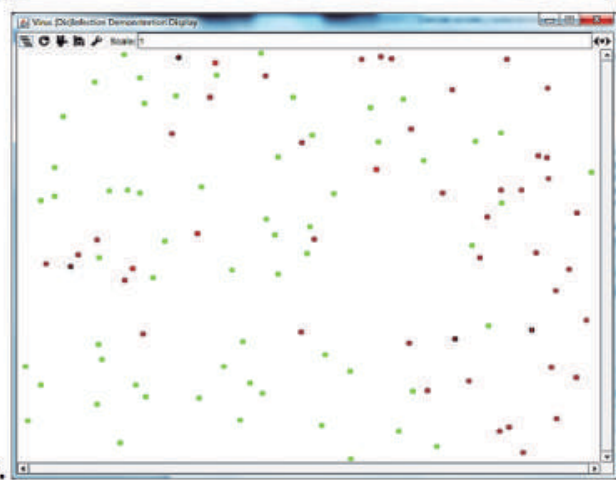

Consola
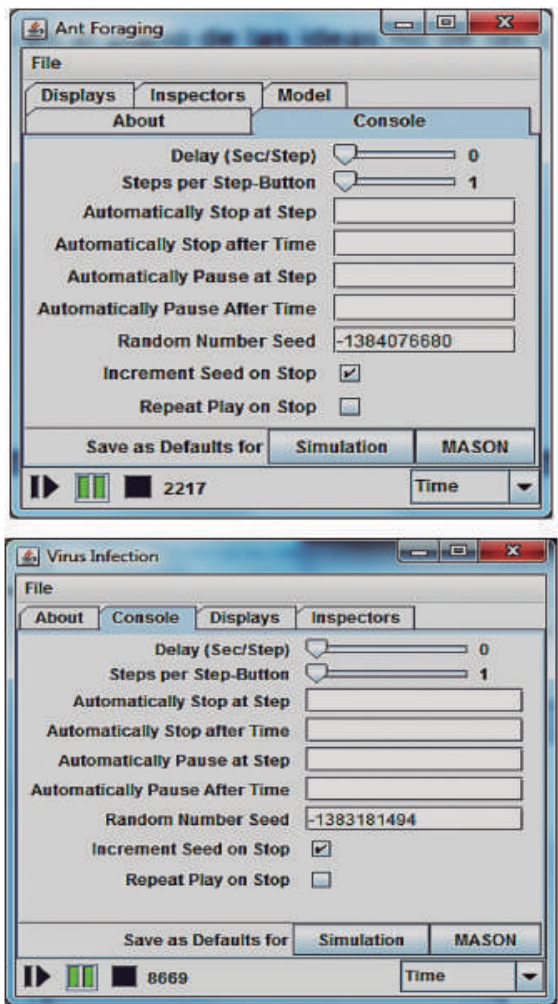

Figura 1. A. Modelo de dos hormonas (hormigas forajidas); B. Modelo de infeccion viral. Fuente: ejecutado por el autor con Mason v.19. (UMLet v.14.2)

En la figura 1 se presentan dos problemas de radical diferencia, pero que pueden ser modelados bajo parámetros similares. El modelo $A$ de las hormigas forajidas y el modelo $B$ de infección viral. En el primero las hormigas artificiales se desplazan del nido para buscar alimento 
sobrepasando obstáculos y realizando recorridos de acuerdo al nivel de feromonas depositadas de modo gradual en el nido y los alimentos. En el segundo modelo un virus (partículas rojas) infecta una población (partículas verdes), las cuales pueden ser desinfectadas por las partículas sanas (rojas con negro). En ambos casos opera un principio de aleatoriedad, que hace que los agentes (hormigas y partículas) se muevan en diferentes direcciones. Las interacciones sociales producen, además, procesos de desequilibrio en el accionar nervioso de los agentes (aunque sigan las reglas de un sistema de producción). Si se refinan los parámetros y se calibran los elementos que configuran los modelos se pueden obtener resultados que desafían el sentido común. Por esta razón la simulación resulta útil en la comprensión de las dinámicas sociales de modo ontológico disimiles entre sí.

Los modelos de simulación pueden describir en términos sistémicos la cognición de los agentes y una cognición agencial; es decir el microcomportamiento y el comportamiento de los actores y su agenciamiento convertido en macrocomportamiento sobre el nivel de la organización social o comunitaria. Esta perspectiva se conoce como bottom-up (de abajo hacia arriba) dada la naturaleza emergente y autoorganizativa de su formalización. Por supuesto que los agentes son representaciones formales o elementos de un conjunto, los cuales se influencian a través de interacciones como ocurre en la realidad con los organismos vivos. De este modo se pueden visualizar y microvalidar cadenas de causación identificadas mediante la simulación con la finalidad de encontrar posibles explicaciones a las conexiones que aparecen como resultado (Moss and Edmonds, 2005).

Wilensky y Rand (2015) realizaron una revisión teórica de los MBA's, en general, la cual incluye sus aplicaciones a la cuestión urbana. Debido a que la principal característica de los MBA's es su dinamismo, sus aplicaciones generalmente se enfocan en la planificación. Desde los inicios de los 90 cuando el auge de los MBA's llegó a las ciencias sociales del lado de la física, la biología y la ingeniería; sin duda, la idea de esta modelización explotó inspirada por el modelo de segregación expuesto a principios de los 70 por Thomas Schelling (1978) cuando planteó para algunos el primer MBA.

Algunas técnicas mezclan diagramas de modelos de lenguaje unificado MLU y MBA's. Forrester et al. (2014) realizaron la simulación del sistema complejo socioecológico (Complex social-ecological systems -SES-) de la costa de Kenia. Realizaron trabajo de campo en la zona con pescadores y demás pobladores para después codificar el modelo en la plataforma NetLogo. El resultado es la creación de un MLU, que opera como mapa cognitivo de este sistema social rural, en el que se puede 
describir el producto de las simulaciones realizadas a través de un MBA². La validación del modelo se realizó de modo posterior con los propios agentes y se pudo establecer aspectos importantes para los tomadores de decisiones, realizadores de políticas, en niveles multiescala con afinidad entre la disminución de la pobreza y la trascendencia del ecosistema.

Podemos decir que este tipo de trabajos establecen dilucidar las interacciones entre la aparición de fenómenos emergentes, la conservación de lo biodiverso y la administración de los recursos. Una perspectiva sustentable a partir de este tipo de ejercicios implica un plano demostrativo de problemáticas y la necesidad de posibles dinámicas organizativas para buscar soluciones. Tal vez no sorprenda que las simulaciones multiagentes en la investigación del manejo de los ecosistemas permita conceptualizar un desarrollo sostenible donde el conocimiento local y el contexto sean trascendentales (Bousquet and Le Page, 2004). Booch, Rumbaugh y Jacobson (2005) presentan un interesante análisis sobre la relación entre los MLU y los MBA; proponen cómo los MLU son, al lado de los pseudocódigos, eficientes modelos conceptuales igual de rigurosos y eficaces a los códigos computacionales. Su intención es explicar a los lectores de cualquier área el funcionamiento de un sistema a través de la combinación de aspectos gráficos y lenguaje natural para describir el modelo planteado (Wilensky and Rand, 2015).

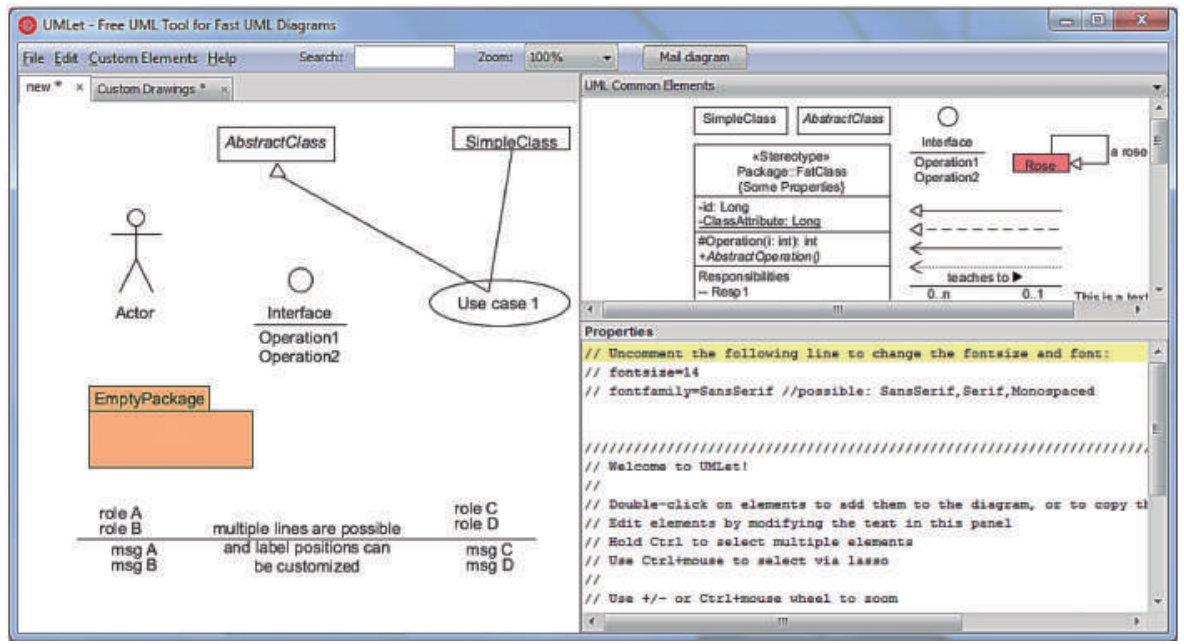

Figura 2. Creación de un MUL.

Fuente: ejecutado por el autor usando UMLet.

\footnotetext{
${ }^{2}$ El modelo y sus detalles se pueden consultar en la siguiente dirección Web: http://www.espa.ac.uk/.
} 


\section{Tecnologías interdisciplinares de investigación social}

\section{Algoritmos genéticos (AG) y algoritmos culturales (AC)}

Los algoritmos de la complejidad van más allá del modelado por el modelado. La receta interdisciplinaria, a este respecto, se compone de diferentes ingredientes metodológicos. Algunos recetarios complejos se abastecen de las metaheurísticas, por ejemplo: el AG para estudiar los mercados de valores artificiales (Chen and Yeh, 2002) a través de series temporales (LeBaron, Arthur and Palmer, 1999), redes neuronales artificiales (LeBaron, 2002), redes sociales (Borgatti and Everett, 1999), sistemas sociales autoorganizados (Klüver, 1996) o pequeñas poblaciones (Reeves, 1993) entre muchas otras aplicaciones. Las referencias de la incumbencia de los AG's para las ciencias sociales se cuentan por centenares.

Para encontrar el sabor, el estudioso decidido puede profundizar en el trabajo práctico estas técnicas disponibles para el paladar investigativo. Un algoritmo genético, creado por John Holland, es un procedimiento evolutivo de búsqueda de optimización mediante la simulación de un proceso de selección de una nueva generación a partir de una actual para encontrar rendimiento óptimo: (i) se crea una población aleatoria de soluciones o individuos (por ejemplo, binarios); (ii) se busca una solución de la mejor adecuación o fitness; (iii) nacen descendientes o se generan las mejores soluciones puntuadas; (iv) se utiliza un método de selección (por ejemplo, selección por torneo) con un umbral aleatorio, la más alta puntuación deviene padre o madre; (v) uno o dos pares son elegidos, con dos padres hay recombinación sexual y con uno clonación; (vi) se puede establecer mutación (cambiar sus dígitos); (vii) los pasos dos y tres se deben repetir hasta encontrar la solución que mejor se ajuste al problema planteado. Se conoce como un método de optimización para problemas que se presentan como irresolubles (Holland, 1995).

El AC creado por Robert Reynolds basado en premisas similares a las de la evolución y sus mecanismos de selección natural — tal como ocurrió con los algoritmos genéticos de Holland- se implementa en un modelo de evolución cultural como mínimo en dos niveles evolucionarios: micro y macro (Reynolds, 1994). A grosso modo, el AC se compone de tres partes: (i) un área en la que se define la población; (ii) un área de creencias en las que la población decide creer, para dar paso a un protocolo comunicativo donde se desarrolla la interacción que conduce al crossover y (iii) un protocolo de comunicación. Un AC es un modelo computacional derivado de la metáfora de la selección natural y sus mecanismos evolutivos, respecto al espacio de creencias de una población. El algoritmo estudia cómo las creencias de 
una población interactúan con el conocimiento compuesto de cinco tipos: topográfico, histórico, normativo, situacional y del dominio técnico ${ }^{3}$.

Los dos modelos son lo que se denominan modelos adaptativos; realizados para implementar el aprendizaje en tanto habilidad, no solo humana. Se les conoce como optimización dado que la población evoluciona cuando se optimizan los agentes, en una adecuación colectiva y una población adaptada (Suleiman, Klaus and Gilbert, 2000). La programación genética se especializa en la búsqueda de rendimiento; por esta razón ambos algoritmos se los utiliza en computación como métodos de optimización.

Diferentes aspectos de la programación evolutiva promueven la modelización de problemas sociales de diferente índole. Uno de los tantos campos en los que la programación evolutiva resulta útil se conoce como antropología evolutiva; orientada hacia la genética de poblaciones, los múltiples modelos de evolución cultural, los problemas de la transmisión y replicación cultural, la innovación, la cooperación, la vida y sociedades artificiales y la evolución de los modelos cognitivos de sistemas orgánicos. Reynolds y Kotbi (2003) presentaron diferentes simulaciones, utilizando algunas que combinan modelos multiagentes y algoritmos culturales. El logro de esta propuesta reside en la capacidad de implementar de modo simultáneo los rasgos individuales y las creencias grupales como un proceso derivado de la metáfora de la naturaleza y modelos de matemática biológica.

\section{Sistemas de producción}

Otros métodos centralizados en la computación y las dinámicas de arquitectura de modelado para las ciencias sociales son los sistemas de producción, la microsimulación o los modelos de aprendizaje (Suleiman, Klaus and Gilbert, 2000). Desde las ciencias cognitivas los sistemas de producción operan dentro del campo de la arquitectura cognitiva basada en la resolución de un problema: al seguir un conjunto de reglas de condicionamiento y accionar, una memoria de trabajo y un intérprete de las reglas. Las reglas contienen dos fases: (i) una fase condicionante, cuando la

\footnotetext{
${ }^{3}$ Para Reynolds y Kobti (2003) estas cinco categorías básicas del conocimiento cultural solidifican el espacio de creencias de cualquier tipo de modelo de evolución cultural. Esta configuración establece lo que se denomina un espacio de creencias el cual inicia con la aceptación, luego el cambio y se interrelaciona con los cinco tipos de conocimiento para ejercer la influencia social. En el modelo formal cada una de estas partes contiene un formalismo computacional en el que se basan las variables de la simulación; además, en este contexto, cada tipo de conocimiento es una posible solución al problema inicialmente planteado. Así, cada tipo de conocimiento es una forma o planteamiento para resolver aquel problema; y cada uno tiene una estructura específica difícil de explicar en este breve espacio.
} 
regla se activa y (ii) una fase de acción, que determina que ocurre cuando la regla se activa. El intérprete determina cuando una de estas reglas entra en acción y considera las condiciones de su encuentro. Una aplicación práctica de un sistema de producción son los agentes que conforman los MBA's.

El modelo de producción con mayor difusión es conocido como SOAR (John, Vera and Newell, 1994; Newell, 1990) y opera a la manera de una arquitectura o máquina de aprendizaje. Se usó para el procesamiento semántico y sus contextos, la toma de decisiones y la comprensión de rangos del performance humano a partir de pruebas de tipo estímulorespuesta, tipificación y razonamiento silogístico. Kim, Lerch y Simon (1995) utilizaron el SOAR para la exploración de problemas de la representación interna a través de investigaciones sobre el uso de la imaginería mental en la búsqueda. Por ejemplo, la inferenciación no proposicional o lo que se conoce metarazonamiento (razonar sobre la razón, por ejemplo: controlar la deliberación de un objeto-nivel, deliberar o actuar). El SOAR también se usó en el modelado de agentes individuales en organizaciones.

\section{Redes alimentarias}

Otras aplicaciones se presentan, quizá, más útiles en otros niveles de la organización y complejidad social debido a su sencillez y disponibilidad de plataformas; además de la no necesidad de codificación computacional. Tanto las redes como los patrones figuran en los conglomerados de organismos vivos en diferentes escalas. La vieja sistémica propuso diferentes formas de comprender los seres vivos, von Bertalanffy (1976) - el padre de la teoría general de sistemas - consideraba que la teoría de redes, información, autómatas y juegos hacían parte de la teoría de sistemas en sus tendencias contemporáneas. Como veremos muchos cientistas sociales tomaron en serio estas y otras premisas a partir de las nociones clásicas de las ciencias sistémicas y la complejidad. Más allá de los modelos matemáticos de enorme dificultad, algunas de las ideas mejor adaptadas a la realidad social parten de principios básicos, formalismos claramente explicados y aplicaciones prácticas a nivel técnico.

A pesar de que la práctica de diagramar de redes alimentarias o tejidos de relaciones es bastante antigua como la antropología misma, se presume que hacia 1880 el herpetólogo y entomólogo italiano Lorenzo Camerano (1856-1917) realizó las primeras exploraciones a este respecto. Aunque fue hasta 1912 cuando aparecieron las primeras redes formales de insectos, predadores y parásitos que se alimentan del algodón realizadas por Pierce, Cushman y Hood (1912) (Egerton, 2007; Pascual and Dunne, 2006; Wells, 2013). Estos diagramas no son lo que conocemos ahora como ARS, 
pero presentan una fuerte correlación con la técnica al menos en el plano formal de establecer tipos de relaciones semejantes y considerar los pesos de esas relaciones. La correspondencia se establece entre las posibilidades de identificar redes alimentarias y de abastecimiento para organizaciones en contextos rurales o urbanos. La estructura de estas redes permite conocer parte de las dinámicas poblacionales de muchos grupos sociales, su estructura de conformación y sus características de sostenibilidad. El campo es denominado como redes ecológicas y se adhiere a los principios de los estudios ecológicos, la antropología alimentaria (Harris, 1985) y la sociología del alimento y comida (Murcott, 1983).

En una importante recopilación adelantada por el Santa Fe Institute —SFI-, Pascual y Dunne (2006) presentaron una serie de trabajos sobre redes ecológicas a través de los lentes de la complejidad. El trabajo presentado por Cartozo, Garlaschelli y Caldarelli (2006) presenta una explicación completa de las redes alimentarias a la luz de la moderna teoría de grafos con un rubro pedagógico sobre los diferentes significados y aplicaciones de los algoritmos empleados en campos como las redes formadas por relaciones de depredación en comunidades ecológicas. A partir de topologías, coeficientes de conglomerados, distancias, grados de distribución, conectividad, relaciones alométricas (crecimiento), optimización de los modelos de nicho, de cascada y de red mundial se busca obtener resultados a través de la aplicación de teoría de grafos sobre aspectos tales como la universalidad de este tipo de redes, los fenómenos de emergencia, la sostenibilidad de las redes ecológicas y sus propiedades.

Una de las técnicas posibles dentro de este tipo de redes, es el anidado. Este concepto proviene del campo de la biogeografía de las islas implementada por Atmar y Paterson (1993) para conocer la distribución de un agregado de animales entre un conjunto espacial de islas. El anidado y sus métricas se utilizan como un medio posible de conocer la estructura subyacente a la relación animales-plantas y su red mutua. Bascompte y Jordana (2006) consideran que el anidamiento $(N)$ puede ser definido como $N=(100-\mathrm{T}) / 100$ con un valor de rango que gira entre 0 y 1 . Por medio de estos valores asignados se genera una matriz binaria con la finalidad de hallar las propiedades relevantes entre redes de animales-plantas. Algunos de estos algoritmos utilizan modelos nulos en los cuales cada célula de la matriz (similar a la de un AC) tiene la misma probabilidad de ser ocupada "una probabilidad estimada como la fracción del total de las interacciones" (Bascompte and Jordana, 2006, p. 149). Las relaciones son halladas a través del análisis de paquetes de matrices, diversas métricas de anidamiento y 
modelos nulos; y los resultados son grados de heterogeneidad entre especies, distribuciones de las conexiones entre especies o conocer si la estructura de una red ecológica es menos o más cohesiva a partir de generalidades.
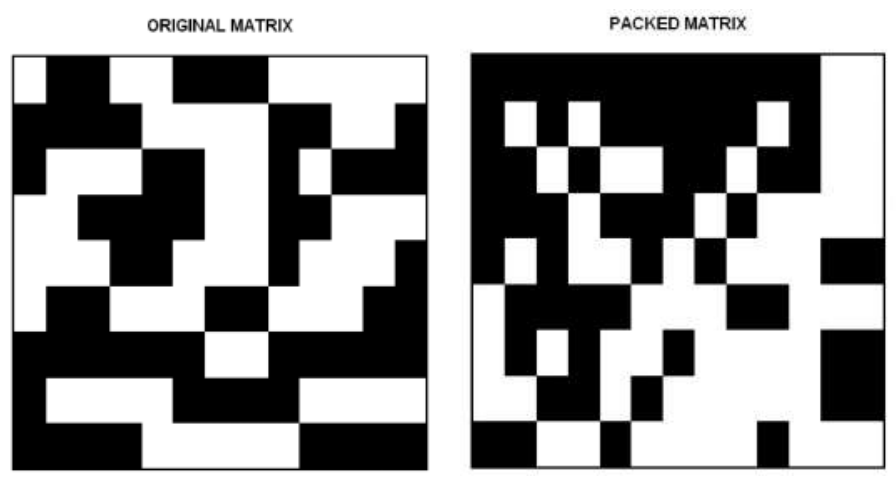
abcdefghijk $7 \mathrm{~m}$
A0110011100000
B1111000011001
C1000110010111
D0011110011000
E0001100010001
F0110001100011
G1111110011111
H1000011110000
I1111000001111

Figura 2. Análisis de anidado.

Fuente: ejecutado por el autor usando NeD.

La figura 2 muestra una comunidad cualquiera de animales y plantas agrupadas en dos hileras con valores binarios y letras. Se pueden observar el cúmulo de las interacciones y el grado de anidamiento. En un caso de comparación estos valores establecen diferenciaciones y similitudes, grados de complejidad o los mecanismos de interés en la generación de patrones derivados de la naturaleza (tal como se observa en los datos arrojados en el análisis).

Otros trabajos recientes emplean modelos de redes sostenibles aplicadas a poblaciones de agricultores. Hace pocos meses el equipo liderado por el antropólogo Stephen Lansing presentó un reporte sobre Bali (Indonesia), en donde un juego de simulación evolutivo puede demostrar el feedback (retroalimentación) entre las milenarias terrazas de arroz realizadas por los granjeros de la zona y sus decisiones y la ecología de los arrozales. El punto central del trabajo es la explicación de cómo se articula una transición de lo local a lo global (Lansing et al., 2017) sobre todo cuando un SC exhibe un proceso de autoorganización. El modelo, además, explica como la cooperación multiescala desde la comunidad hasta el nivel de las líneas divisoras de aguas se mantiene a través de los siglos de modo sostenible. Tiempo atrás Lansing (2006) había propuesto que los patrones fractales de plantación mantienen cosechas óptimas sin control central. 
Aunque el desarrollo de este trabajo es de carácter rural, las implicaciones sobre lo urbano son trascendentales pero difíciles de tratar en este espacio ${ }^{4}$.

\section{Sistemas multiagentes (MAS)}

En el campo de la sociología de las organizaciones algunos modelos proponen formalizaciones multiagentes de la acción organizada aplicadas al campo de la sostenibilidad organizacional de cualquier tipo de entidad social. En la figura 3 se observa una práctica rutinaria con la plataforma SocLab (Sibertin-Blanc et al., 2013).
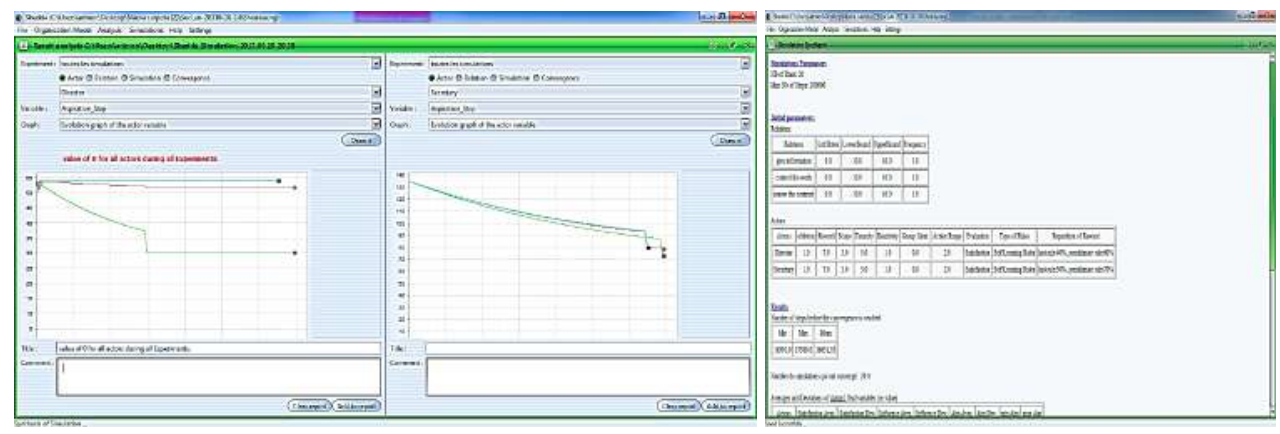

Figura 3. Simulación de modelo organizacional.

Fuente: ejecutado por el autor usando SocLab.

El recorrido de la izquierda equivale a la trayectoria del director y el del centro al recorrido realizado por la secretaria, en la derecha se muestra los datos generales de la simulación. El metamodelo establece unas relaciones o estados (b_min, b_max, movimiento) entre actores (capacidad/ poder/satisfacción/influencia) hacia la solidaridad (niveles), estados (b_min, b_max, movimiento), entre constreñimientos (on_bmin, on_bmax) de dependencia (participación/impacto, efecto). Permite estudiar también los movimientos de cada uno de los actores, los niveles de solidaridad y diversos tipos de análisis configurando los valores asignados a cada actor. Es posible aplicar el método a diferentes poblaciones rurales o urbanas y desarrollar los estudios de juegos de cooperación desde otro punto de vista complementario al de la antropología evolutiva o los estudios de economía social.

\footnotetext{
${ }^{4}$ Un análisis de este caso respecto a las cuestiones políticas del modelo y la sostenibilidad de la comunidad de Bali respecto a la intromisión de actores externos en este ecosistema y sus problemáticas derivadas se encuentra en Reynoso (2013).
} 
El estudio de los sistemas complejos y su comprensión propone diversas perspectivas. Uno de los metamodelos recientes es el Simulating Knowledge Dynamics in Innovation Networks - SKIN- (Gilbert, Ahrweiler and Pyka, 2010). Esta plataforma es una posibilidad de modelar bajo un cuantioso número de variables, la implementación de sistemas multiagentes. El modelo fue aplicado a diferentes estudios de toma de decisiones, relaciones y estructuras de redes de innovación. Gilbert, Ahrweiler y Pyka (2014) editaron todo un volumen donde exponen las particularidades del modelo, algunos hallazgos, aplicaciones e implicaciones a nivel metodológico. Aunque el modelo es aplicado al campo de la dinámica industrial a nivel organizativo, su objetivo es identificar las propiedades de redes de innovación y sus dinámicas de cara al conocimiento de redes intencionales.

\section{Estimaciones fractales, sintaxis espacial (SE) y autómatas celulares (AC's)}

El campo de las estimaciones fractales, asociado a las métricas de la sintaxis espacial y modelado basado en $\mathrm{AC}^{\prime}$ s, es abundante. Debido a que las simulaciones basadas en $\mathrm{AC}^{\prime}$ 's arrojan patrones fractales como resultado, la sintaxis espacial estudia la lógica espacial rural o urbana y su estructura adyacente; estas tecnologías poseen un poderoso aspecto grafico básico para la comprensión de la accesibilidad, visibilidad y los tipos de espacios (públicos, residenciales, urbanos, rurales). Diversos investigadores suelen mezclar las técnicas, algoritmos, plataformas de trabajo, presupuestos conceptuales, premisas cognitivas y una compleja red teórica en el estudio de lo urbano y lo rural de manera interdisciplinar (Amorim, Barros and Cruz, 2014; Ariza-Villaverde, Jiménez-Hornero and Ravé, 2013; Batty 2013; Chen and Feng, 2012; Chen and Wang, 2013; Feng and Chen, 2010; Frankhauser, 2015; Salingaros, 2005). Se puede decir que cualquier ortodoxia movilizada en este campo pierde su tiempo en el debate de la espacialidad contemporánea. 
A.

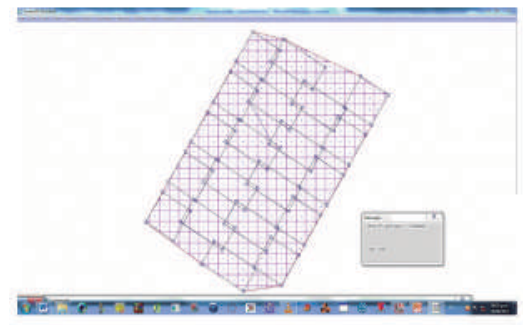

C.

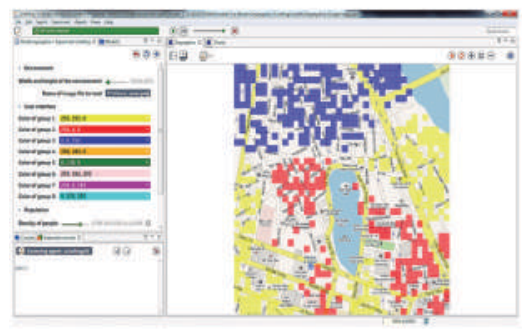

B.

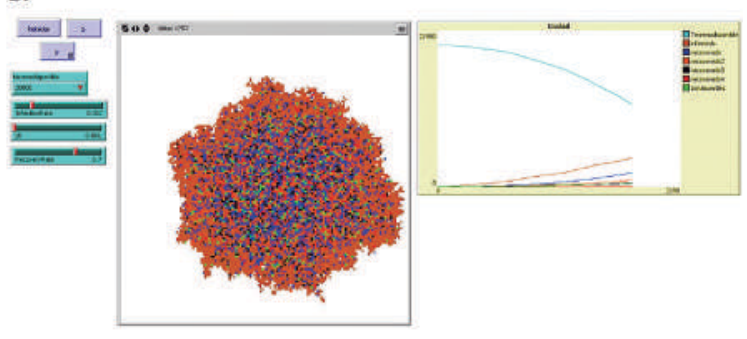

D.

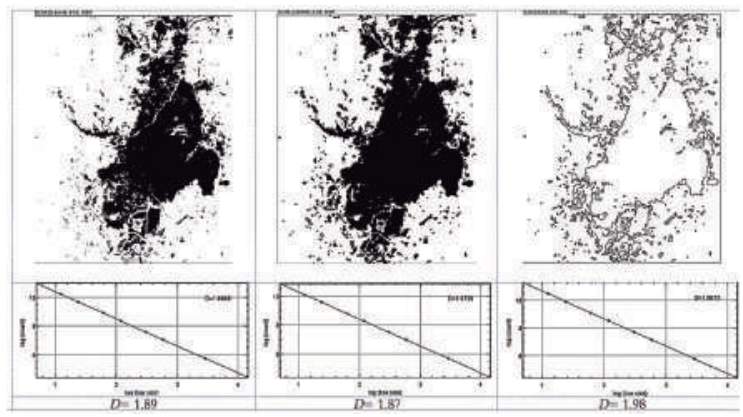

Figura 4. A. Análisis espacial con SE; B. Diseño de una ciudad artificial utilizando AC's en NetLogo; C. Modelo de segregación de Schelling utilizando AC's en GAMA; D. Análisis de dimensión fractal con diferentes algoritmos de morfología matemática en ImageJ. Fuente: imágenes A, B y D ejecutadas por el autor, imagen C ejecutada por el autor usando GAMA Platform v1.7.0. ImageJ v.1.51h.

Al lado de los sistemas de funciones iteradas (SFI), los sistemas y las gramáticas recursivas (tipo L-Systems), la modelización de fractales de difusión y la teoría de la ciudad fractal, Batty (2013) creó un campo transdisciplinar fructífero en las investigación de espacialidades urbanas y rurales desde fines de los 80 . Con un alto potencial de aplicación a la planeación y las problemáticas derivadas de la sostenibilidad su perspectiva conjunta se diversificó desde el ensamblaje de la fractalidad, los AC's, la SE y de modo reciente con el ARS, al partir de tecnologías de sistemas georreferenciados y el estudio de las imágenes de sensoramiento remoto.

Las estimaciones fractales se proponen como tecnologías apropiadas para la resolución de problemas sobre la sostenibilidad de las ciudades y las implicaciones negativas de los efectos del fenómeno de la dispersión o sprawl y las consecuencias para los espacios rurales que confluyen. Frankhauser (2015) presenta un panorama amplio respecto a los múltiples ámbitos de estudio en los cuales la fractalidad es un indicador y presupuesto teórico 
sobre el campo de la sostenibilidad socioespacial. Batty (2013) configuró lo que llama un nuevo paradigma del estudio de las ciudades y la ruralidad donde, para generar espacios geográficos más apropiados, estos deben ser densos para ser más sostenibles.

El hallazgo del crecimiento de las ciudades fue realizado por Brand (2010) que — en sus estudios denominados "ciencia de la tierra entera" estableció que las interacciones sociales más eficientes requieren espacios geopoblacionales con mayores densidades, contrario a los espacios que tienden a continuar el sprawl urbano. Según Brand (2010) las altas densidades permiten mejores condiciones de transporte, reducción del sprawl y construcciones costo-efectivas mejores.

Las CDC y sus potenciales algorítmicos parten del estudio de entornos más sostenibles, su crecimiento, tamaño, rango, redes jerárquicas, estructuras, sintaxis espaciales, distancia de y entre redes complejas, crecimiento fractal y las simulaciones urbanas como algunas de las posibilidades de aplicación práctica durante el siglo XX y de cara a un futuro sostenible. Si consideramos los objetivos de desarrollo sostenible y su interacción, las prácticas de modelado referidas en este espacio proporcionan valiosos mecanismos de trabajo pragmático de cara a la globalización, los entornos, las acciones y decisiones que procuran sostenibilidad.

Tabla 2. Posibilidades tecnológicas, modelos y tipos de complejidad aplicadas al estudio de lo rural y lo urbano desde las CDC

\begin{tabular}{lll}
\hline Tecnologías & Tipos de modelos & Tipo de complejidad \\
\hline Redes & $\begin{array}{l}\text { Complejos, cognitivos, } \\
\text { no-lineales, caóticos, } \\
\text { estructurales. }\end{array}$ & Organizada/no-lineal \\
\hline Autómatas celulares (AC's) & $\begin{array}{l}\text { Aleatorios, estocásticos, } \\
\text { autoorganizados. }\end{array}$ & Aleatoria/estocástica \\
\hline $\begin{array}{l}\text { Modelos basados en agentes } \\
\text { (MBA's) }\end{array}$ & $\begin{array}{l}\text { Espaciales, cognitivos, } \\
\text { predictivos, emergentes, } \\
\text { autoorganizados. }\end{array}$ & Organizada \\
\hline $\begin{array}{l}\text { Modelos de sistemas multiagentes } \\
\text { (MAS) }\end{array}$ & $\begin{array}{l}\text { Espaciales, cognitivos, } \\
\text { predictivos, emergentes, } \\
\text { autoorganizados. }\end{array}$ & Organizada \\
\hline $\begin{array}{l}\text { Modelos de lenguaje unificado } \\
\text { (MLU) }\end{array}$ & $\begin{array}{l}\text { Cognitivos, } \\
\text { computacionales } \\
\text { (diagramas). }\end{array}$ & Organizada \\
\hline La dinámica de sistemas (DS) & $\begin{array}{l}\text { Estadísticos, emergentes, } \\
\text { autoorganizados. }\end{array}$ & Organizada \\
\hline
\end{tabular}




\begin{tabular}{lll}
\hline Algoritmos genéticos (AG's) & $\begin{array}{l}\text { Aleatorios, estocásticos, } \\
\text { genéticos, optimización. }\end{array}$ & $\begin{array}{l}\text { Organizada/aleatoria/ } \\
\text { estocástica }\end{array}$ \\
\hline Algoritmo cultural (AC) & $\begin{array}{l}\text { Aleatorios, estocásticos, } \\
\text { genéticos, optimización. }\end{array}$ & $\begin{array}{l}\text { Organizada/aleatoria/ } \\
\text { estocástica }\end{array}$ \\
\hline Fractalidad & $\begin{array}{l}\text { Recursivos, aleatorios, } \\
\text { estocásticos, deterministas, } \\
\text { caóticos. }\end{array}$ & Caótica/no-lineal \\
\hline Sistemas de producción & $\begin{array}{l}\text { Cognitivos, } \\
\text { computacionales. }\end{array}$ & Organizada \\
\hline Neurociencias sociales & $\begin{array}{l}\text { Estadísticos, cognitivos, } \\
\text { experimentales, } \\
\text { longitudinales. }\end{array}$ & Organizada \\
\hline
\end{tabular}

Fuente: elaboración propia por parte del autor.

En la tabla 2 se presenta una descripción provisional de los tipos de tecnologías abordadas en este espacio y sus posibles modelos, algunos con una precisión difícil de describir en este espacio debido a las especificidades de cada tecnología y su background teórico. Los tipos de complejidad orientan la comprensión de cada técnica y su campo de realización, lejos de cualquier dogmatismo respecto a estas posibilidades cada vez observamos como diferentes interdisciplinas, transdisciplinas o colectivos mixtos consideran el estudio de los entornos de vida humanos y no-humanos como una prioridad en sí. Dada la necesidad de establecer puentes entre diferentes presupuestos teóricos, metodológicos y epistemológicos, la causalidad correlativa vista desde la medida y el territorio nos dice que los contextos si importan.

\section{Conclusión. ¿Por qué modelos de complejidad para el estudio intrincado de las ruralidades y lo urbano contemporáneo?}

Contrario a lo que podría suponerse, las tecnologías de investigación social propuestasen esteespacioy susartíficessealejan delexceso de confianza como constante epistemológica. Estas son alternativas metodológicas para enfrentar los problemas que conocemos en la contemporaneidad: la no linealidad de los sistemas biológicos; la autocriticabilidad; la emergencia; la complejidad de los sistemas humanos y su adaptación; las transiciones de fase de los sistemas sociales en sus espacios físicos; la no-normalidad en las distribuciones poblacionales entre muchos otros. El espacio es corto para explorar otras alternativas eficaces en la búsqueda de sostenibilidades de 
diferente índole, las técnicas, métodos y estudios expuestos contribuyen al conocimiento de lo social, rural y urbano de manera decidida.

Nilsson et al. (2016) recuerdan que las dimensiones económicas, sociales y del entorno están cruzadas por la interacción de los contextos. Esta perspectiva de sostenibilidad implica reconocer las interacciones como algo vital en la consecución de los objetivos de poblaciones más sostenibles. El nexo entre investigadores, comunidades, tomadores de decisiones y políticas públicas reclama sinergias y formas coherentes de relacionamiento al reconsiderar las clásicas asimetrías del poder. Las ciencias sociales de cara a estas necesidades pueden aportar demasiado si el nivel de sus resultados revela realidades posibles bajo perspectivas multidimensionales como las aquí expuestas.

\section{Referencias bibliográficas}

Amorim, L.M.E., Barros, M.N.M. and Cruz, D. (2014). Urban texture and space configuration: An essay on integrating socio spatial analytical techniques. Cities, 39, 58-67.

Ariza-Villaverde, A.B., Jiménez-Hornero, F.J. and Ravé, E.G.D. (2013). Multifractal analysis of axial maps applied to the study of urban morphology. Comput Environ Urban Systems, 38, 1-10.

Atmar, W. and Patterson, B.D. (1993). The Measure of Order and Disorder in the Distribution of Species in Fragmented Habitat. Oecologa, 96, 373-382.

Batty, M. (2013). The New Science of Cities. Massachusetts, USA: The MIT Press.

Bascompte, J. and Jordana, P. (2006). The Structure of Plant-Animal M utualistic Networks. En M. Pascual and J.A. Dunne (Ed.), Ecological Networks: Linking Structure to Dynamics in Food Webs (pp. 143-159). New York, USA: Oxford University Press.

Booch, G., Rumbaugh, J. and Jacobson, I. (2005). The Unified Modeling Language User's Guide. New York, USA: Addison-Wesley.

Borgatti, S. and Everett, M. (1999). Models of core/periphery structures. Social Networks, 21, 375-395.

Bousquet, F. and Le Page, C. (2004). Multi-agent simulations and ecosystem management: A review. Ecol Modell, 176, 313-332.

Brand, S. (2010). Whole Earth Discipline: Why Dense Cities, Nuclear Power, Transgenic Crops, Restored Wildlands, Radical Science, and Geoengineering are Necessary. New York, USA: Atlantic Books.

Cartozo, C.C., Garlaschelli, G. and Caldarelli, G. (2006). Graph Theory and Food Webs. En M. Pascual and J.A. Dunne (Ed.), Ecological Networks: Linking Structure to Dynamics in Food Webs (pp. 93-117). New York, USA: Oxford University Press.

Chen, S.H. and Yeh, C.H. (2002). On the Emergent Properties of Artificial Stock Markets: The Efficient Market Hypothesis and the Rational Expectations Hypothesis. Journal of Economic Behaviour and Organization, 49, 217-239.

Chen, Y. and Feng, J. (2012). Fractal-based exponential distribution of urban density and self-affine fractal forms of cities. Chaos, Solitons \& Fractals, 45, 1404-1416.

Chen, Y. and Wang, J. (2013). Multifractal characterization of urban form and growth: The case of Beijing. Environment and Planning B: Urban Analytics and City Science, 40, 884-904. 
de Vries, B. and Petersen, A. (2009). Conceptualizing sustainable development: An assessment methodology connecting values, knowledge, worldviews and scenarios. Ecological Economics, 68, 1006-1019.

Egerton, F.N. (2007). Understanding food chains and food webs, 1700-1970. Bulletin of the Ecological Society of America, 88, 50-69.

Feng, J. and Chen, Y. (2010). Spatiotemporal evolution of urban form and land use structure in Hangzhou, China: Evidence from fractals. Environment and Planning B: Urban Analytics and City Science, 37, 838-856.

Forrester, J. et al. (2014). Modeling Social-Ecological Problems in Coastal Ecosystems: A Case Study. Complexity, 19, 73-82.

Frankhauser, P. (2015). From Fractal Urban Pattern Analysis to Fractal Urban Planning Concepts. En M. Helbich, J.J. Arsanjani and M. Leitner (Ed.), Computational Approaches for Urban Environments (pp. 13-48). Geneva, Switzerland: Springer International Publishing.

Gilbert, N. and Troitzsch, K.G. (2005). Simulation for the Social Scientist. Buckingham, United Kingdom: Open University Press.

Gilbert, N., Ahrweiler, P. and Pyka, A. (2010). The SKIN (Simulating Knowledge Dynamics in Innovation Networks) model. Mainz, Germany: Johannes Gutenberg University Mainz, University of Hohenheim.

Gilbert, N., Ahrweiler, P. and Pyka, A. (Ed.). (2014). Simulating Knowledge Dynamics in Innovation Networks. Berlin, Germany: Springer-Verlag.

Harris, M. (1985). Good to Eat: Riddles of Food and Culture. New York, USA: Simon \& Schuster.

Higgins, A.J. et al. (2010). Applying operations research to agricultural value chain to achieve a balance in efficiency and resilience. Journal of the Operations Research Society, 61, 964973.

Holland, J. (1995). Hidden Order: How Adaptation Builds Complexity. Reading, England: Addison-Wesley.

ICSU. (2016). A Draft Framework for Understanding SDG Interactions. Recuperado de https://icsu.org/cms/2017/05/SDG-interactions-working-paper.pdf.

John, B.E., Vera, A.H. and Newell, A. (1994). Toward real-time GOMS: A model of expert behavior in a highly interactive task. Behavior and Information Technology, 13, 255-267.

Kim, J., Lerch, F. and Simon, H.A. (1995). Internal representation and rule development in object-oriented design. ACM Transactions on Computer-Human Interaction, 2 (4), 357-390.

Klüver, J. (1996). Simulations of Self Organizing Social Systems. En F. Faulbaum and W. Bandilla (Ed.), SoftStat 95. Advances in Statistical Software (pp. 425-432). Stuttgart, Germany: Lucius.

Lansing, J.S. (2006). Perfect Order: Recognizing Complexity in Bali. New Jersey, USA: Princeton University Press.

Lansing, J.S. et al. (2017). Adaptive self-organization of Bali's ancient rice terraces. Proceedings of the National Academy of Sciences, 114 (25), 6504-6509.

LeBaron, B. (2002). Short Memory Traders and Their Impact on Group Learning in Financial Markets. Proceedings of the U.S. National Academy of Sciences, 99, 7201-7206.

LeBaron, B., Arthur, W.B. and Palmer, R. (1999). Time Series Properties of an Artificial Stock Market. Journal of Economic Dynamics and Control, 23, 1487-1516.

Moss, S. and Edmonds, B. (2005). Sociology and Simulation: Statistical and Qualitative Cross-Validation. AJS, 110 (4), 1095-1131.

Murcott, A. (Ed.). (1983). The Sociology of Food and Eating: Essays on the Sociological Significance of Food. Aldershot, England: Gower. 
Newell, A. (1990). Unified Theories of Cognition. Cambridge, USA: Harvard University Press.

Nilsson, M., Griggs, D. and Visbeck, M. (2016). Map the interactions between Sustainable Development Goals. Nature, 534, 320-322.

Northrop, R.B. and Connor, A.N. (2013). Ecological Sustainability. Understanding Complex Issues. Boca Raton, USA: CRC Press, Taylor \& Francis Group.

Pascual, M. and Dunne, J.A. (Ed.). (2006). Ecological Networks: Linking Structure to Dynamics in Food Webs. New York, USA: Oxford University Press.

Pierce, W.D., Cushman, R.A. and Hood, C.E. (1912). The insect enemies of the cotton boll weevil. U.S. Department of Agriculture, Bureau of Entomology Bulletin, 100, 1-99.

Poincaré, H. (1908). Science et Méthode. Paris, France: Flammarion.

Reeves, C.R. (1993). Using genetic algorithms with small populations. En S. Forrest (Ed.), Proceedings of the Fifth International Conference on Genetic Algorithms, University of Illinois at Urbana-Champaign (pp. 92-99). San Mateo, USA: Morgan Kaufmann.

Reynolds, G.R. (1994). An Introduction to Cultural Algorithms. Recuperado de http://ai.cs.wayne.edu/ai/availablePapersOnLine/IntroToCA.pdf.

Reynolds, R. and Kobti, Z. (2003). A Multi-Agent Simulation Using Cultural Algorithms: The Effect of Culture on the Resilience of Social Systems. Recuperado de http://ieeexplore. ieee.org/document/1299917/?reload=true.

Reynoso, C. (2006). Complejidad y caos: una exploración antropológica. Buenos Aires, Argentina: Editorial SB.

Reynoso, C. (2013). Etnicidad y redes territoriales: perspectivas de complejidad. En B. Nates (Coord.), La frontera, las fronteras: diálogos transversales en estudios territoriales contemporáneos (pp. 63-90). Riohacha, Colombia: RETEC.

Salingaros, N.A. (2005). Principles of Urban Structure. Amsterdam, Netherlands: Techne Press. Schelling, T. (1978). Micromotives and Macrobehavior. New York, USA: Norton.

Sibertin-Blanc, C. et al. (2013). SocLab: A Framework for the Modeling, Simulation and Analysis of Power in Social Organizations. Journal of Artificial Societies and Social Simulation, 16 (4). Recuperado de http://jasss.soc.surrey.ac.uk/16/4/8.html.

Suleiman, R., Troitzsch, K.G. and Gilbert, N. (Ed.). (2000). Tools and Techniques for Social Science Simulation. Heidelberg, Germany: Physica-Verlag.

Tufte, E.R. (1997). Visual Explanations. Images and Quantities, Evidence and Narrative. Cheshire/ Connecticut, USA: Graphics Press.

von Bertalanffy, L. (1976). Teoría general de los sistemas. Fundamentos, desarrollo, aplicaciones. Buenos Aires, Argentina: Fondo de Cultura Económica.

Wells, J. (2013). Complexity and Sustainability. New York, USA: Routledge.

Wilensky, U. and Rand, W. (2015). An Introduction to Agent-Based Modeling. Modeling Natural, Social, and Engineered Complex Systems with NetLogo. Cambridge, USA: The MIT Press. 